\title{
Western Blot
}

National Human Genome Research Institute (NHGRI)

\section{Source}

National Human Genome Research Institute (NHGRI). Western Blot.

Western blotting is a laboratory technique used to detect a specific protein in a blood or tissue sample. The method involves using gel electrophoresis to separate the sample's proteins. The separated proteins are transferred out of the gel to the surface of a membrane. The membrane is exposed to an antibody specific to the target protein. Binding of the antibody is detected using a radioactive or chemical tag. A western blot is sometimes used to diagnose disease. 